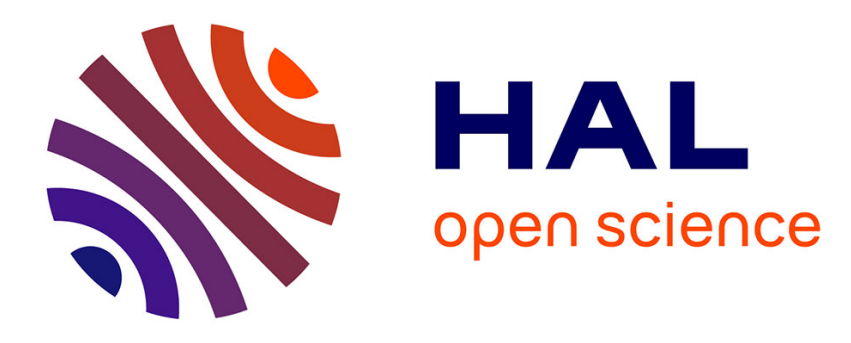

\title{
Scattering of electromagnetic waves by rough dielectric surfaces \\ J.-J. Greffet
}

\section{To cite this version:}

J.-J. Greffet. Scattering of electromagnetic waves by rough dielectric surfaces. Physical Review B: Condensed Matter and Materials Physics (1998-2015), 1988, 37 (11), pp.6436-6441. 10.1103/PhysRevB.37.6436 . hal-01617143

\section{HAL Id: hal-01617143 \\ https://hal-iogs.archives-ouvertes.fr/hal-01617143}

Submitted on 16 Oct 2017

HAL is a multi-disciplinary open access archive for the deposit and dissemination of scientific research documents, whether they are published or not. The documents may come from teaching and research institutions in France or abroad, or from public or private research centers.
L'archive ouverte pluridisciplinaire HAL, est destinée au dépôt et à la diffusion de documents scientifiques de niveau recherche, publiés ou non, émanant des établissements d'enseignement et de recherche français ou étrangers, des laboratoires publics ou privés. 


\title{
Scattering of electromagnetic waves by rough dielectric surfaces
}

\author{
J.-J. Greffet \\ Laboratoire d'Energetique Moleculaire et Macroscopique Combustion, Ecole Centrale, \\ Centre National de la Recherche Scientifique, Grande Voie Des Vignes, 92295 Chatenay-Malabry Cédex, France
}

(Received 29 July 1987)

\begin{abstract}
We present a treatment of the scattering of electromagnetic waves at rough dielectric surfaces using both the Rayleigh hypothesis and the extinction theorem. An explicit expression relating $s$ - and $p$-polarized components of the field in the dielectric with the incident field is given. A Dyson equation is then derived for the field and an exact iterative series solution is given for both transmitted and reflected fields. In order to evaluate quantitatively the accuracy of this method, scattering by a sinusoidal grating is examined in detail; the results are in agreement with rigorous approaches previously reported.
\end{abstract}

\section{INTRODUCTION}

This paper deals with the interaction of an electromagnetic wave with a rough dielectric surface. More specifically we consider the problem of scattering by a determined surface for any case of polarization. This problem has been treated using integral equations and perturbative techniques in Refs. 1-4. The extinction theorem, also coupled with perturbative approaches has been used later. ${ }^{5-8}$ An alternative technique consists of assuming that the Rayleigh expansion is valid and then using the extinction theorem as a boundary condition. This approach has been compared with the simple Rayleigh theory of Toigo et al. for the case of a $p$-polarized wave. ${ }^{9}$ Later an iterative series solution for the problem of the scattering of a $p$-polarized wave by a grating was given by Maradudin ${ }^{10}$ within the same framework. Finally, an integral equation for the reflected field and for any state of polarization was reported by Brown et al. ${ }^{11}$

In the present paper, we assume the validity of the Raleigh hypothesis. The discussion of this point is out of the scope of this paper; for a review of both the theoretical and the practical utility of the Rayleigh ansatz the reader is referred to Refs. $12-14$. Within this approximation we obtain in Sec. II a basic equation relating the spectral components of the incident and transmitted field for any polarization. Thus, this formalism allows a discussion of the depolarization of the fields. This equation is a generalization of part of the work reported in Ref. 9. In Sec. III we show how to obtain a Dyson equation for the transmitted field. This formulation proves to be well suited to the discussion of the homogeneous problem. In Sec. IV, an explicit iterative series solution is obtained for both the transmitted and the reflected fields for any polarization. In the last section we apply the formalism of Sec. II to the problem of scattering by a grating in order to compare its limits with rigorous solutions.

\section{INTEGRODIFFERENTIAL EQUATIONS FOR THE SCATTERED ELECTROMAGNETIC FIELDS IN A LOCAL LINEAR MEDIUM}

We consider an electromagnetic wave of arbitrary polarization impinging on a rough dielectric surface charac- terized by the equation

$$
z=S(\rho), \quad \rho=(x, y) .
$$

The medium occupies the lower half-space $[z<S(\rho)]$ (Fig. 1). We assume that the medium is homogeneous, isotropic, and has a local, linear susceptibility $\chi(\omega)=[\epsilon(\omega)-1] / 4 \pi$ where $\epsilon(\omega)$ is the complex dielectric constant. The circular frequency of the incident radiation is $\omega$. The field in the medium will be noted $\mathbf{E}_{t}$ (transmitted) whereas the field outside the medium will be the sum of the incident field $\mathbf{E}_{i}$ and the scattered field $\mathbf{E}_{r}$. For all the fields we assume the temporal dependence $\exp (-i \omega t)$.

The transmitted electric field must satisfy the Helmholtz equation:

$$
\nabla^{2} \mathbf{E}_{t}+\epsilon k_{0}^{2} \mathbf{E}_{t}=0 \text {, }
$$

where $k_{0}=\omega / c$ and $c$ is the light velocity in the vacuum.

Assuming that the Rayleigh expansion is valid, one may write $\mathbf{E}_{t}$ in the following form:

$$
\mathbf{E}_{t}(\mathbf{r})=\int d \boldsymbol{\kappa} \mathbf{e}_{t}(\boldsymbol{\kappa}) \exp \left[i\left(\boldsymbol{\kappa} \cdot \boldsymbol{\rho}-\gamma_{t} z\right)\right],
$$

where

$$
\gamma_{t}^{2}+\kappa^{2}=\epsilon k_{0}^{2} \text { and } \operatorname{Im}\left(\gamma_{t}\right)>0 .
$$

Similar representations may be introduced for the incident and scattered fields:

$$
\mathbf{E}_{i}(\mathbf{r})=\int d \kappa \mathbf{e}_{i}(\kappa) \exp \left[i\left(\kappa \cdot \rho-\gamma_{i} z\right)\right],
$$

where

$$
\begin{aligned}
& \gamma_{i}^{2}+\kappa^{2}=k_{0}^{2} \text { and } \gamma_{i}=k_{0} \cos \theta_{i} \\
& \mathbf{E}_{r}(\mathbf{r})=\int d \kappa \mathbf{e}_{r}(\kappa) \exp \left[i\left(\boldsymbol{\kappa} \cdot \boldsymbol{\rho}+\gamma_{r} z\right)\right],
\end{aligned}
$$

where

$$
\gamma_{r}^{2}+\kappa^{2}=k_{0}^{2} \text { and } \operatorname{Im}\left(\gamma_{r}\right)>0 .
$$

Note that (2.3) may be viewed as a modal expansion of $\mathbf{E}_{t}$ since each spectral component satisfies (2.2).

In order to obtain the coefficients $e_{t}(\kappa)$ we will use the extinction theorem which appears as a boundary condi- 
tion (for a discussion of this point see Refs. 15 and 16).

$$
\begin{aligned}
\mathbf{E}_{i}(\mathbf{r})=-\frac{1}{4 \pi k_{0}^{2}} \nabla \times \nabla \times \int_{S} & \mid \mathbf{E}_{t}\left(\mathbf{r}^{\prime}\right) \frac{\partial G\left(\mathbf{r}, \mathbf{r}^{\prime}\right)}{\partial n^{\prime}} \\
& \left.-G\left(\mathbf{r}, \mathbf{r}^{\prime}\right) \frac{\partial \mathbf{E}_{t}\left(\mathbf{r}^{\prime}\right)}{\partial n^{\prime}}\right] d S^{\prime},
\end{aligned}
$$

where $\mathbf{r}$ lies in the medium, $\mathbf{r}^{\prime}$ is a typical point on $S$, $\partial / \partial n^{\prime}$ denotes differentiation along the outward normal to $S$, and

$$
\boldsymbol{G}\left(\mathbf{r}, \mathbf{r}^{\prime}\right)=\frac{\exp \left(i k_{0}\left|\mathbf{r}-\mathbf{r}^{\prime}\right|\right)}{\left|\mathbf{r}-\mathbf{r}^{\prime}\right|}
$$

designates the free-space Green's function.

In principle the transmitted field may be found by inverting (2.9). The scattered field is then deduced from

$$
\begin{aligned}
\mathbf{E}_{r}(\mathbf{r})=\frac{1}{4 \pi k_{0}^{2}} \boldsymbol{\nabla} \times \boldsymbol{\nabla} \times \int_{S} & \left(\mathbf{E}_{t}\left(\mathbf{r}^{\prime}\right) \frac{\partial G\left(\mathbf{r}, \mathbf{r}^{\prime}\right.}{\partial n^{\prime}}\right. \\
& \left.-G\left(\mathbf{r}, \mathbf{r}^{\prime}\right) \frac{\partial \mathbf{E}_{t}\left(\mathbf{r}^{\prime}\right)}{\partial n^{\prime}}\right) d S^{\prime} .
\end{aligned}
$$

To determine the spectral amplitudes $\mathbf{e}_{t}(\boldsymbol{\kappa})$ we substitute (2.3) in (2.9). To this purpose, it is also convenient to introduce in (2.9) the spectral representation of the freespace Green's function $G$ :

$$
G\left(\mathbf{r}, \mathbf{r}^{\prime}\right)=\frac{i}{2 \pi} \int \frac{d \boldsymbol{\kappa}}{\gamma_{r}} \exp \left[i \boldsymbol{\kappa} \cdot\left(\rho-\boldsymbol{\rho}^{\prime}\right)+i \gamma_{r}\left|z-z^{\prime}\right|\right] .
$$

Moreover, the following relations allow us to perform the integral in (2.9) over the $x-y$ plane:

$$
\begin{aligned}
& \frac{\partial}{\partial n^{\prime}}=\left(\hat{\mathbf{n}}^{\prime} \cdot \nabla\right) \text { with } \hat{\mathbf{n}}^{\prime}=\frac{1}{\left[1+\left(\nabla_{\|} S\right)^{2}\right]^{1 / 2}}\left(-\nabla_{\|} S, 1\right), \\
& d S^{\prime}=d x^{\prime} d y^{\prime}\left[1+\left(\nabla_{\|} S\right)^{2}\right]^{1 / 2} .
\end{aligned}
$$

Integrating by parts using the identity

$$
\begin{aligned}
\nabla_{\|}\{\exp & {\left.\left[i\left(\boldsymbol{\kappa}^{\prime}-\boldsymbol{\kappa}\right) \cdot \boldsymbol{\rho}^{\prime}+i\left(\gamma_{r}-\gamma_{t}^{\prime}\right) \boldsymbol{S}\left(\boldsymbol{\rho}^{\prime}\right)\right]-1\right\} } \\
= & i\left[\boldsymbol{\kappa}^{\prime}-\boldsymbol{\kappa}+\left(\gamma_{r}-\gamma_{t}^{\prime}\right) \boldsymbol{\nabla}_{\|} \boldsymbol{S}\right] \\
& \times \exp \left[i\left(\boldsymbol{\kappa}^{\prime}-\boldsymbol{\kappa}\right) \cdot \boldsymbol{\rho}^{\prime}+i\left(\gamma_{r}-\gamma_{t}^{\prime}\right) S\left(\boldsymbol{\rho}^{\prime}\right)\right],
\end{aligned}
$$

one obtains after some algebra an integral equation for the transmitted field

$$
\mathbf{e}_{i}(\boldsymbol{\kappa})=\frac{\epsilon-1}{4 \pi} \frac{1}{2 \pi \gamma_{r}} \mathbf{k}_{r}^{-} \times \mathbf{k}_{r}^{-} \times \int d \boldsymbol{\kappa}^{\prime} \mathbf{e}_{t}\left(\boldsymbol{\kappa}^{\prime}\right) I\left(\kappa, \kappa^{\prime}\right),
$$

where the scattering potential $I\left(\kappa, \boldsymbol{\kappa}^{\prime}\right)$ is

$$
\begin{aligned}
I\left(\boldsymbol{\kappa}, \boldsymbol{\kappa}^{\prime}\right)=\int & d \boldsymbol{\rho}^{\prime} \exp \left[i\left(\boldsymbol{\kappa}^{\prime}-\boldsymbol{\kappa}\right) \cdot \boldsymbol{\rho}^{\prime}\right] \\
& \times \frac{\exp \left[i\left(\gamma_{r}-\gamma_{t}^{\prime}\right) S\left(\boldsymbol{\rho}^{\prime}\right)\right]}{\gamma_{r}-\gamma_{t}^{\prime}},
\end{aligned}
$$

where $\gamma$ is a function of $\boldsymbol{\kappa}$ and $\gamma^{\prime}$ a function of $\boldsymbol{\kappa}^{\prime}$, and

$$
\mathbf{k}_{r, t}^{ \pm}=\left(\boldsymbol{\kappa}, \pm \gamma_{r, t}\right) \text {. }
$$

Note that the Rayleigh hypothesis is invoked for the transmitted field only. If we are interested in the reflected field outside the selvedge region, (2.7) constitutes an exact representation. Since Eq. (2.16) yields each spectral component of the transmitted field, the reflected field may be computed outside the selvedge region without any additional hypothesis.

Before solving the integral equation (2.16), we will introduce a local basis (i.e., depending on $\kappa$ ) in order to treat separately the $s$ and $p$ components of the field. Assuming $\boldsymbol{\nabla} \cdot \mathbf{E}_{t}=0$ we may take advantage of the transverse nature of the field to write the spectral components in the following form:

$$
\mathbf{e}_{t}(\boldsymbol{\kappa})=e_{t s}(\boldsymbol{\kappa}) \widehat{\mathbf{a}}_{s}\left(\mathbf{k}_{t}^{-}\right)+e_{t p}(\boldsymbol{\kappa}) \hat{\mathbf{a}}_{p}\left(\mathbf{k}_{t}^{-}\right),
$$

where $\hat{\mathbf{a}}_{s}\left(\mathbf{k}_{t}^{-}\right)$and $\hat{\mathbf{a}}_{p}\left(\mathbf{k}_{t}^{-}\right)$are two unit vectors perpendicular to $\mathbf{k}_{t}^{-}$defined as follows:

$$
\begin{aligned}
& \hat{\mathbf{a}}_{s}\left(\mathbf{k}_{t}^{-}\right)=\hat{\mathbf{z}} \times \hat{\boldsymbol{\kappa}}, \\
& \hat{\mathbf{a}}_{p}\left(\mathbf{k}_{t}^{-}\right)=\hat{\mathbf{a}}_{s}\left(\mathbf{k}_{t}^{-}\right) \times \frac{\mathbf{k}_{t}^{-}}{\left|\mathbf{k}_{t}^{-}\right|} .
\end{aligned}
$$

The vectors $\left(\hat{\mathbf{a}}_{p}\left(\mathbf{k}_{t}^{-}\right), \hat{\mathbf{a}}_{s}\left(\mathbf{k}_{t}^{-}\right), \mathbf{k}_{t}^{-}\right)$form a right-handed coordinate system. The coefficients $e_{t s}$ and $e_{t p}$ are the $s$ and $p$ components of the field. Using this basis the double vectorial product which appears as a linear operator referred as $R\left(\kappa, \kappa^{\prime}\right)$ hereafter may be written in a matrix form:

$$
R\left(\boldsymbol{\kappa}, \boldsymbol{\kappa}^{\prime}\right)=-k_{0}^{2}\left(\begin{array}{cc}
\hat{\boldsymbol{\kappa}} \cdot \hat{\boldsymbol{\kappa}}^{\prime} & -\frac{\gamma_{t}^{\prime}}{n k_{0}} \hat{\boldsymbol{\kappa}}^{\prime} \cdot(\hat{\mathbf{z}} \times \hat{\boldsymbol{\kappa}}) \\
-\frac{\gamma_{r}}{\boldsymbol{\kappa}_{0}} \hat{\boldsymbol{\kappa}} \cdot\left(\hat{\mathbf{z}} \times \hat{\boldsymbol{\kappa}}^{\prime}\right) & \frac{\boldsymbol{\kappa} \boldsymbol{\kappa}^{\prime}+\left(\hat{\boldsymbol{\kappa}} \cdot \hat{\boldsymbol{\kappa}}^{\prime}\right) \gamma_{r} \gamma_{t}^{\prime}}{n k_{0}^{2}}
\end{array}\right) .
$$

Finally we obtain a new form of the integral equation (2.16) for the $s$ and $p$ components of the transmitted field:

$$
\left[\begin{array}{l}
e_{i s}(\boldsymbol{\kappa}) \\
e_{i p}(\boldsymbol{\kappa})
\end{array}\right]=\frac{\epsilon-1}{4 \pi} \frac{1}{2 \pi \gamma_{r}} \int d \boldsymbol{\kappa}^{\prime} I\left(\boldsymbol{\kappa}, \boldsymbol{\kappa}^{\prime}\right) R\left(\boldsymbol{\kappa}, \boldsymbol{\kappa}^{\prime}\right)\left[\begin{array}{l}
e_{t s}\left(\mathbf{k}^{\prime}\right) \\
e_{t p}\left(\boldsymbol{\kappa}^{\prime}\right)
\end{array}\right] \text {. }
$$

Equation (2.22) is an integral equation for the transmitted field. Note that the only restriction to its validity depends on the Rayleigh hypothesis. In the following section we will modify $(2.22)$ in order to separate contributions to the scattered fields due to either the bulk or the selvedge region. Then, we will derive a Dyson equation for the transmitted field. In Sec. IV, we show that the ex- 
act solution of Eq. (2.22) may be expressed in the form of an iterative matricial series solution generalizing the results of Lopez et al. ${ }^{17}$ for the scalar case and of Maradudin for the $p$-polarized wave incident on a grating. ${ }^{10}$

\section{A DYSON EQUATION FOR THE TRANSMITTED FIELD}

Let us now consider the transmitted field as the superposition of the zero-order solution (corresponding to a flat interface) and a correction term due to the selvedge region. We introduce the following notations:

$$
\mathbf{e}_{t}(\kappa)=\mathbf{e}_{t}^{(0)}(\kappa)+\mathbf{e}_{t}^{(1)}(\kappa) .
$$

Let us consider the case of a flat interface $[S(\rho)=0]$. From (2.17) we deduce:

$$
I\left(\boldsymbol{\kappa}, \boldsymbol{\kappa}^{\prime}\right)=\frac{4 \pi^{2}}{\gamma_{r}-\gamma_{t}} \delta\left(\boldsymbol{\kappa}-\boldsymbol{\kappa}^{\prime}\right)
$$

Introducing (3.2) in (2.22) we readily obtain the Fresnel transmission coefficients for the amplitude of the fields:

$$
\left[\begin{array}{l}
e_{t s}^{(0)}(\boldsymbol{\kappa}) \\
e_{t p}^{(0)}(\boldsymbol{\kappa})
\end{array}\right]=\left(\begin{array}{cc}
\frac{2 \gamma_{r}}{\gamma_{r}+\gamma_{t}} & 0 \\
0 & \frac{2 n \gamma_{r}}{\epsilon \gamma_{r}+\gamma_{t}}
\end{array}\right)\left[\begin{array}{l}
e_{i s}(\boldsymbol{\kappa}) \\
e_{i p}(\boldsymbol{\kappa})
\end{array}\right)
$$

where we have used the following identities:

$$
\begin{aligned}
& (\epsilon-1) k_{0}^{2}=\left(\gamma_{t}-\gamma_{r}\right)\left(\gamma_{t}+\gamma_{r}\right), \\
& \left(\kappa^{2}+\gamma_{r} \gamma_{t}\right)\left(\gamma_{r}+\gamma_{t}\right)=\left(\epsilon \gamma_{r}+\gamma_{t}\right) k_{0}^{2} .
\end{aligned}
$$

To proceed it is convenient to substitute the decomposition

$$
I\left(\boldsymbol{\kappa}, \boldsymbol{\kappa}^{\prime}\right)=\frac{4 \pi^{2}}{\gamma_{r}-\gamma_{t}} \delta\left(\boldsymbol{\kappa}-\boldsymbol{\kappa}^{\prime}\right)+J\left(\boldsymbol{\kappa}, \boldsymbol{\kappa}^{\prime}\right),
$$

where the first term on the right-hand-side member appears as the contribution of the bulk, whereas the second is due to the selvedge region. Introducing (3.1) and (3.5) in (2.22) one obtains a Dyson equation for $\mathbf{e}_{t}(\boldsymbol{\kappa})$ :

$$
\left[\begin{array}{l}
e_{t s}(\boldsymbol{\kappa}) \\
e_{t p}(\boldsymbol{\kappa})
\end{array}\right]=\left[\begin{array}{l}
e_{t s}^{(0)}(\boldsymbol{\kappa}) \\
e_{t p}^{(0)}(\boldsymbol{\kappa})
\end{array}\right]-\frac{\gamma_{r}-\gamma_{t}}{4 \pi^{2}} R^{-1}(\boldsymbol{\kappa}, \boldsymbol{\kappa}) \int d \boldsymbol{\kappa}^{\prime} R\left(\boldsymbol{\kappa}, \boldsymbol{\kappa}^{\prime}\right) J\left(\boldsymbol{\kappa}, \boldsymbol{\kappa}^{\prime}\right)\left[\begin{array}{l}
e_{t s}\left(\boldsymbol{\kappa}^{\prime}\right) \\
e_{t p}\left(\boldsymbol{\kappa}^{\prime}\right)
\end{array}\right] \text {. }
$$

This formulation proves to be well suited for the treatment of the homogeneous problem. Let the incident field be null: (3.6) then reduces to

$$
\left[\begin{array}{l}
e_{t s}(\boldsymbol{\kappa}) \\
e_{t p}(\boldsymbol{\kappa})
\end{array}\right]=-\frac{\gamma_{r}-\gamma_{t}}{4 \pi^{2}} R^{-1}(\boldsymbol{\kappa}, \boldsymbol{\kappa}) \int d \boldsymbol{\kappa}^{\prime} R\left(\boldsymbol{\kappa}, \boldsymbol{\kappa}^{\prime}\right) J\left(\boldsymbol{\kappa}, \boldsymbol{\kappa}^{\prime}\right)\left[\begin{array}{l}
e_{t s}\left(\boldsymbol{\kappa}^{\prime}\right) \\
e_{t p}\left(\boldsymbol{\kappa}^{\prime}\right)
\end{array}\right] .
$$

This equation is a good starting point in investigations of the dispersion relation of the polaritons. This subject is not pursued here since it has been studied by means of a similar approach in Refs. 18 and 19.

On the other hand, (3.6) is useful in deriving accurate approximations for the transmitted field. Note that $J\left(\kappa, \kappa^{\prime}\right)$ appears as a scattering potential whose depth is related with the amplitude $h$ of the function $S(\rho)$ and with a range of the order of the autocorrelation length $l$ for $J(0, \kappa)$. From these physical remarks it appears that a Born approximation should provide a good solution for the transmitted field for surfaces having low ratio $l / h$. Note that this condition is consistent with the Rayleigh hypothesis. In the following section we turn to the problem of establishing an exact iterative series solution for the field.

\section{AN ITERATIVE SERIES SOLUTION FOR THE FIELDS}

In this section we solve Eq. (2.22) by expanding the fields and the function $I\left(\kappa, \kappa^{\prime}\right)$ as follows:

$$
\begin{aligned}
& \mathbf{e}_{t}(\boldsymbol{\kappa})=\sum_{n=0}^{\infty} \frac{\mathbf{e}_{t}^{(n)}(\boldsymbol{\kappa})}{n !}, \\
& I\left(\boldsymbol{\kappa}, \boldsymbol{\kappa}^{\prime}\right)=\sum_{n=0}^{\infty} \frac{(i)^{n}\left(\gamma_{r}-\gamma_{t}^{\prime}\right)^{n-1}}{n !} S^{n}\left(\boldsymbol{\kappa}^{\prime}-\boldsymbol{\kappa}\right),
\end{aligned}
$$

with

$$
S^{n}\left(\boldsymbol{\kappa}^{\prime}-\boldsymbol{\kappa}\right)=\int d \boldsymbol{\rho}^{\prime} \exp \left[i\left(\boldsymbol{\kappa}^{\prime}-\boldsymbol{\kappa}\right) \cdot \boldsymbol{\rho}^{\prime}\right] S^{n}\left(\boldsymbol{\rho}^{\prime}\right) .
$$

Introducing (4.1) and (4.2) in (2.22) and equating each term of same order one obtains

$$
\left[\begin{array}{l}
e_{t s}^{(n)}(\boldsymbol{\kappa}) \\
e_{t p}^{(n)}(\boldsymbol{\kappa})
\end{array}\right]=\frac{\gamma_{t}-\gamma_{r}}{4 \pi^{2}} R^{-1}(\boldsymbol{\kappa}, \boldsymbol{\kappa}) \int d \boldsymbol{\kappa}^{\prime} R\left(\boldsymbol{\kappa}, \boldsymbol{\kappa}^{\prime}\right) \sum_{q=1}^{n}\left(\begin{array}{l}
n \\
q
\end{array}\right)(i)^{q}\left(\gamma_{r}-\gamma_{t}^{\prime}\right)^{q-1} S^{q}\left(\boldsymbol{\kappa}^{\prime}-\boldsymbol{\kappa}\right)\left[\begin{array}{l}
e_{t s}^{(n-q)}\left(\boldsymbol{\kappa}^{\prime}\right) \\
e_{t p}^{(n-q)}\left(\boldsymbol{\kappa}^{\prime}\right)
\end{array}\right] \quad(n \text {th order }) \text {. }
$$

Equation (4.4) gives the well-known perturbation expressions for $q=1$ for both polarizations. $1,2,4,9,11$ From the examination of the nondiagonal terms of $R$ one also obtains the classical result that the crossed terms (i.e., the depolarization) appears only to second order. Finally, expression (4.4) specialized to the $p$-polarized case is the same as (3-28-b) of Ref. 10 apart from differences in notations. 
The scattered field is computed using $(2.10)-(2.15)$ as in Sec. II:

$$
\mathbf{e}_{r}(\boldsymbol{\kappa})=\frac{\epsilon-1}{4 \pi} \frac{1}{2 \pi \gamma_{r}} \mathbf{k}_{r}^{+} \times \mathbf{k}_{r}^{+} \times \int d \boldsymbol{\kappa}^{\prime} \mathbf{e}_{t}\left(\boldsymbol{\kappa}^{\prime}\right) \int d \rho^{\prime} \exp \left[i\left(\boldsymbol{\kappa}^{\prime}-\boldsymbol{\kappa}\right) \cdot \boldsymbol{\rho}^{\prime}\right] \frac{\exp \left[-i\left(\gamma_{r}+\gamma_{t}^{\prime}\right) S\left(\rho^{\prime}\right)\right]}{\gamma_{r}+\gamma_{t}^{\prime}}
$$

Let us introduce the operator $P\left(\kappa, \kappa^{\prime}\right)$ which essentially performs the projection of $e_{t}(\kappa)$ on the basis $\left(\hat{\mathbf{a}}_{s}\left(\mathbf{k}_{r}^{+}\right), \hat{\mathbf{a}}_{p}\left(\mathbf{k}_{r}^{+}\right)\right)$:

$$
P\left(\boldsymbol{\kappa}, \boldsymbol{\kappa}^{\prime}\right)=-k_{0}^{2}\left(\begin{array}{cc}
\hat{\boldsymbol{\kappa}} \cdot \hat{\boldsymbol{\kappa}}^{\prime} & \frac{-\gamma_{t}^{\prime}}{n k_{0}} \hat{\boldsymbol{\kappa}}^{\prime} \cdot(\hat{\mathbf{z}} \times \hat{\boldsymbol{\kappa}}) \\
\frac{\gamma_{r}}{k_{0}} \hat{\boldsymbol{\kappa}} \cdot\left(\hat{\mathbf{z}} \times \boldsymbol{\kappa}^{\prime}\right) & \frac{-\gamma_{r} \gamma_{t}^{\prime}\left(\hat{\boldsymbol{\kappa}}^{\prime} \hat{\boldsymbol{\kappa}}^{\prime}\right)+\kappa \boldsymbol{\kappa}^{\prime}}{n k_{0}}
\end{array}\right) .
$$

Introducing (4.2) and (4.6) in (4.5) one obtains, after expanding $\exp \left[-i\left(\gamma_{r}+\gamma_{t}^{\prime}\right) S\left(\rho^{\prime}\right)\right]$ and identifying the terms of same order,

$$
\left[\begin{array}{l}
e_{r s}^{(n)}(\kappa) \\
e_{r p}^{(n)}(\kappa)
\end{array}\right]=\frac{\epsilon-1}{4 \pi} \frac{1}{2 \pi \gamma_{r}} \int d \kappa P\left(\kappa, \kappa^{\prime}\right) \sum_{q=0}^{n-1}\left[\begin{array}{l}
n \\
q
\end{array}\right](i)^{n-q} S^{n-q}\left(\kappa^{\prime}-\kappa\right)\left(\gamma_{r}+\gamma_{t}^{\prime}\right)^{n-q-1}\left(\begin{array}{l}
e_{r s}^{(q)}\left(\kappa^{\prime}\right) \\
e_{r p}^{(q)}\left(\kappa^{\prime}\right)
\end{array}\right) \quad(n \text {th order })
$$

The zero order yields the Fresnel coefficients for reflection:

$$
\left[\begin{array}{l}
e_{r s}^{(0)}(\boldsymbol{\kappa}) \\
e_{r p}^{(0)}(\boldsymbol{\kappa})
\end{array}\right)=\left(\begin{array}{cc}
\frac{\gamma_{r}-\gamma_{t}}{\gamma_{r}+\gamma_{t}} & 0 \\
0 & \frac{\epsilon \gamma_{r}-\gamma_{t}}{\epsilon \gamma_{r}+\gamma_{t}}
\end{array}\right)\left[\begin{array}{l}
e_{i s}(\boldsymbol{\kappa}) \\
e_{i p}(\boldsymbol{\kappa})
\end{array}\right),
$$

and the first order of (4.7) appears to be the same as expression (2.14) of Ref. 6.

\section{NUMERICAL STUDY OF SCATTERING BY A SINUSOIDAL GRATING}

The aim of this section is to investigate the domain of practical utility of the basic result $(2.22)$ and to show that this integral equation is useful far beyond the limits of perturbative approaches. We treat the standard problem of scattering of a plane wave by a dielectric grating. Let $S(x)=h \cos (2 \pi x / d)$ be the equation of the surface and $\mathbf{E}_{i}$ be the electric field of an $s$-polarized electromagnetic plane wave illuminating the grating. As in Sec. II the dielectric medium lies in the lower half-space and is linear, homogeneous and isotropic. Since the surface is periodic the fields may be expanded in the forms

$$
\begin{aligned}
& e_{i}\left(\kappa_{x}, \kappa_{y}\right)=e_{i} \delta\left(\kappa_{x}-\kappa_{i}\right) \delta\left(\kappa_{y}\right), \\
& e_{t}\left(\kappa_{x}, \kappa_{y}\right)=\sum_{n=-\infty}^{+\infty} e_{t n} \delta\left(\kappa_{x}-\kappa_{i}-n \kappa_{g}\right) \delta\left(\kappa_{y}\right), \\
& e_{r}\left(\kappa_{x}, \kappa_{y}\right)=\sum_{n=-\infty}^{+\infty} e_{r n} \delta\left(\kappa_{x}-\kappa_{i}-n \kappa_{g}\right) \delta\left(\kappa_{y}\right),
\end{aligned}
$$

with

$$
\kappa_{g}=\frac{2 \pi}{d}
$$

Introducing these expressions in (2.22) we obtain a linear system:

$$
\gamma_{r n} e_{i} \delta_{q n}=\sum_{n} M_{q n} e_{t n}
$$

with

$$
\begin{aligned}
M_{q n}=\frac{k_{0} \kappa_{g}}{\gamma_{r}} \frac{\epsilon-1}{4 \pi} \int_{0}^{d} & \exp \left[i(q-n) \kappa_{g} x\right] \\
& \times \frac{\exp \left[i\left(\gamma_{r q}-\gamma_{t n}\right) S\left(x^{\prime}\right)\right]}{\gamma_{r q}-\gamma_{t n}} d x^{\prime}
\end{aligned}
$$

and where we have used the following relations:

$$
\begin{aligned}
& \int_{-\infty}^{+\infty} d y^{\prime} \exp \left(i \kappa_{y} y^{\prime}\right)=2 \pi \delta\left(\kappa_{y}\right) \\
& \sum_{q=-\infty}^{+\infty} \exp \left[i q\left(\kappa_{x}-\kappa_{i}\right) d\right]=\kappa_{g} \sum_{q=-\infty}^{+\infty} \delta\left(\kappa_{x}-\kappa_{i}-q \kappa_{g}\right)
\end{aligned}
$$

The transmitted field is obtained by solving this linear system. The reflected field is then deduced from the following relation derived from (4.5) and (5.1):

$$
e_{r}\left(\kappa_{i}+m \kappa_{r}\right)=-\frac{k_{0}^{2} \kappa_{r}}{\gamma_{r m}} \frac{\epsilon-1}{4 \pi} \sum_{n=-\infty}^{+\infty} N_{n m} e_{t n},
$$

where

$$
\begin{aligned}
N_{n m}=\int_{0}^{d} \exp \left[i(n-m) \kappa_{g} x^{\prime}\right] \\
\quad \times \frac{\exp \left[-i\left(\gamma_{r m}+\gamma_{t n}\right) S\left(x^{\prime}\right)\right]}{\gamma_{r m}+\gamma_{t n}} d x^{\prime} .
\end{aligned}
$$


For convenience we will introduce the efficiencies in the $m$ th order for the reflected and the transmitted fields:

$$
\begin{aligned}
& \rho_{m}=\left|\frac{e_{r m}}{e_{i}}\right|^{2} \frac{c_{m}^{r}}{c_{i}}, \\
& \alpha_{m}=\left|\frac{e_{t m}}{e_{i}}\right|^{2} \sqrt{\epsilon} \frac{c_{m}^{t}}{c_{i}},
\end{aligned}
$$

where

$$
\begin{aligned}
& c_{i}=\left[1-\left(\kappa i / k_{0}\right)^{2}\right]^{1 / 2}, \\
& c_{m}^{r}=\left[1-\left[\frac{\kappa_{i}+m \kappa_{g}}{k_{0}}\right]^{2}\right]^{1 / 2}, \\
& c_{m}^{t}=\left[1-\left[\frac{\kappa_{i}+m \kappa_{g}}{\sqrt{\epsilon} k_{0}}\right]^{2}\right]^{1 / 2} .
\end{aligned}
$$

The efficiencies should verify the law of conservation of energy:

$$
\operatorname{Re}\left(\sum_{m}\left(\alpha_{m}+\rho_{m}\right)\right)=1
$$

where only the propagating modes are involved in the sum. This relation will be used to check the results. Details of the numerical calculations are as follows: the integrals in (5.3) and (5.7) are performed with the Simpson quadrature technique. The matrix inversion is performed with the Gauss-Jordan scheme. The matrix is expected to be well conditioned up to a limit value of the ratio $h / d$. Recall that the theoretical limit of validity of the Rayleigh expansion is $h / d=0.072$.

As a first step we present a comparison of the results obtained with the present method, hereafter called modified Rayleigh theory (MRT), with previous calculations by Maystre, ${ }^{20}$ Nevière, Vincent, and Petit, ${ }^{21}$ and Wirgin $^{22}$ using, respectively, a rigorous integral formalism, a rigorous differential formalism, and the approximate Rayleigh theory. Table I shows that, for a grating whose ratio $h / d$ is almost twice the limit value 0.072 , the approximate methods agree well with the reference solutions.

To obtain the limit value of $h / d$ the grating amplitude $h$ is increased. Figure 1 displays the behavior of the sum of the efficiencies which should be equal to 1 according to (5.11). It appears that the energy criterion is satisfied within $1 \%$ for a ratio $h / d$ lower than 0.14 for the partic-

TABLE I. Comparison of M (Maystre result), NVP (Nevière, Vincent, and Petit result), RT (Rayleigh theory), and MRT (modified Rayleigh theory). Total energy $(\mathscr{E})$ and efficiencies $\left(\rho_{n}\right)$ of reflected spectral orders for sinusoidal gratings separating air from a dielectric medium. $\epsilon=2.56, \theta_{i}=26.744^{\circ}, \lambda=0.9$ $\mu \mathrm{m}, d=1 \mu \mathrm{m}, h=0.125 \mu \mathrm{m}$. For RT and MRT the truncation retains 11 terms.

\begin{tabular}{lcccc}
\hline \hline & M & NVP & RT & MRT \\
\hline$\rho_{0}$ & 0.0244 & 0.0244 & 0.0244 & 0.0244 \\
$\rho_{-1}$ & 0.0222 & 0.0222 & 0.0222 & 0.0222 \\
$\mathscr{E}$ & & & 1.000 & 1.000 \\
\hline \hline
\end{tabular}

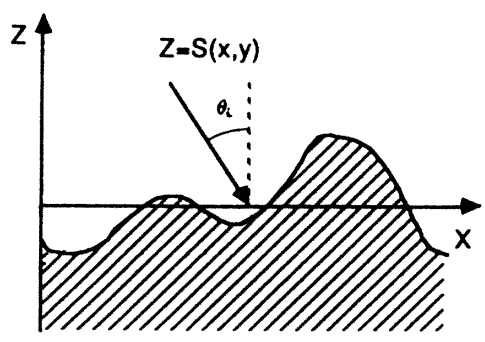

FIG. 1. Scattering geometry.

ular incidence used in the calculation $\left(10^{\circ}\right)$. For larger incidence the behavior of the RT and the MRT is better (see Table I and Ref. 12).

It is worthwhile to compare this method with an alternative approach, namely the Rayleigh theory. An extensive study of the domain of validity of this theory can be found in Ref. 12. Basically, this theory assumes the validity of the Rayleigh expansion for both the reflected and the transmitted fields. Then, the continuity relations are used as boundary conditions for the field. Hence, the limits of this theory are due to the Rayleigh expansion and one may expect that both approaches will fail in the same way when the ratio $h / d$ is increased. However, Fig. 2 shows that the Rayleigh theory has a larger domain of validity than the approach outlined in this section but an oscillatory behavior in the vicinity of the limit of validity.

Next, we examine the behavior of the theory when the amplitude of the corrugation of the surface increases while the ratio $h / d$ remains fixed. Hence, the perturbative treatments fail whereas the Rayleigh hypothesis is still valid. As an example we present in Fig. 3 the results obtained with the MRT for increasing values $h$ up to twice the wavelength. The conclusion is obvious: the integral equation (2.22) has a wider domain of validity than its perturbative solution.

It is shown that the formalism of Sec. II (MRT) leads to very accurate results beyond the theoretical limit of validity. On the other hand no "practical limit" for the

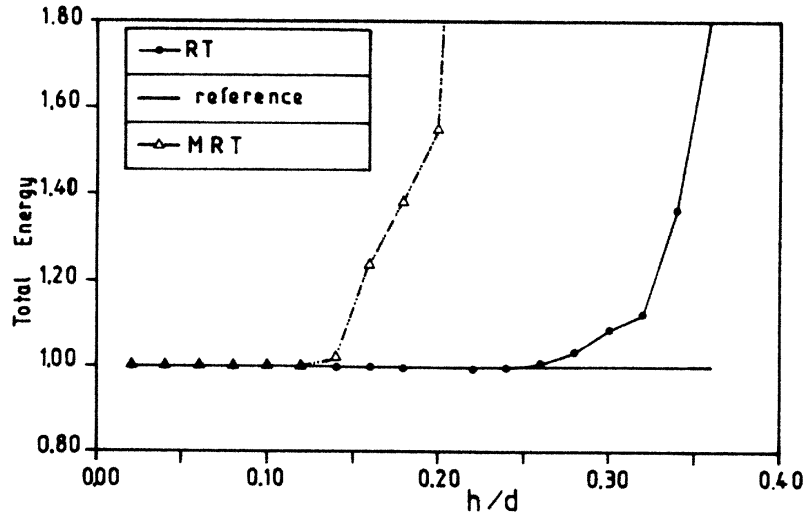

FIG. 2. Total energy computed with the RT and the MRT vs the corrugation amplitude $h / d$. The parameters of the calculation are $\lambda=1 \mu \mathrm{m}, \epsilon=2.1, d=2 \mu \mathrm{m}, \theta_{i}=10^{\circ}$. 


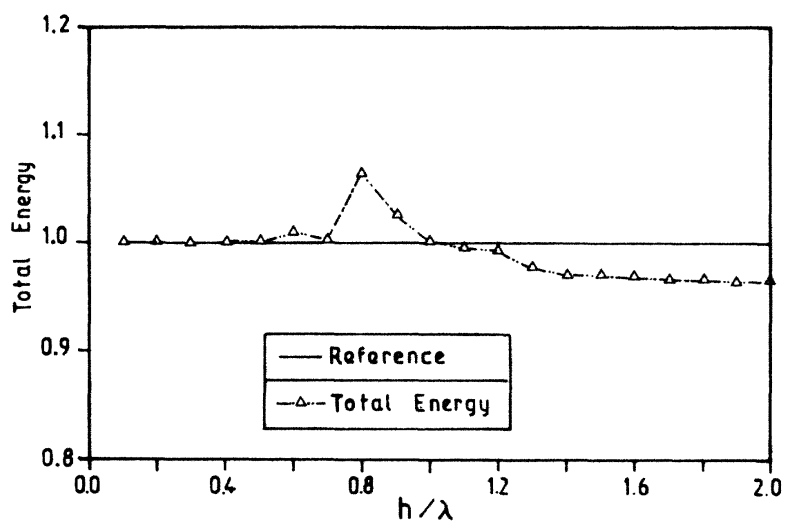

FIG. 3. Total energy computed with the MRT vs the grating amplitude $h$. The parameters are the same as in Fig. 2 .

usefulness of the Rayleigh expansion may be exhibited. Such a limit depends both on the analytical approach involving the Rayleigh expansion (the choice of the boundary condition in the present case) and on the parameters of the problem (i.e., shape of the surface, incidence).

\section{CONCLUSION}

We have presented in this paper a general formulation of scattering of electromagnetic waves at a rough dielectric surface. The use of the Rayleigh approximation leads to a spectral formulation of the problem which proves to be useful. We have shown how it may be used to derive a Dyson equation and, hence, a Born approximation which gives physical insight into the process. Taking advantage of the simplicity of this formulation, we have been able to obtain an explicit iterative series solution to all orders for the scattering of electromagnetic waves on a rough dielectric surface. Finally, we have derived a very simple and accurate numerical scheme for the study of scattering of electromagnetic waves by gratings.

\section{ACKNOWLEDGMENT}

The author thanks Dr. A. Wirgin for helpful discussion.
1J. M. Elson, Phys. Rev. B 12, 2541 (1975).

${ }^{2}$ A. A. Maradudin and D. L. Mills, Phys. Rev. B 11, 1392 (1975).

${ }^{3}$ N. R. Hill, Phys. Rev. B 24, 7112 (1981).

${ }^{4}$ G. S. Agarwal, Phys. Rev. B 14, 846 (1976).

${ }^{5}$ G. S. Agarwal, Opt. Commun. 14, 161 (1975).

${ }^{6}$ G. S. Agarwal, Phys. Rev. B 15, 2371 (1977).

${ }^{7}$ M. Nieto-Vesperinas, J. Opt. Soc. Am. 72, 539 (1982).

${ }^{8}$ D. Winnebrenner and A. Ishimaru, Radio Sci. 20, 161 (1985).

${ }^{9}$ F. Toigo, A. Marvin, V. Celli, and N. R. Hill, Phys. Rev. B 15, 5618 (1977).

${ }^{10}$ A. A. Maradudin, J. Opt. Soc. Am. 73, 759 (1983).

${ }^{11}$ G. C. Brown, V. Celli, M. Haller, and A. Marvin, Surf. Sci. 136, 381 (1984).

${ }^{12}$ A. Wirgin, J. Opt. Soc. Am. 73, 1028 (1983).
${ }^{13}$ A. Wirgin, J. Acoust. Soc. Am. 72, 963 (1982).

${ }^{14}$ R. F. Millar, Radio Sci. 8, 785 (1983), and references therein.

${ }^{15}$ D. N. Pattanayak and E. Wolf, Opt. Commun. 6, 217 (1972).

${ }^{16}$ E. Lalor and E. Wolf, J. Opt. Soc. Am. 62, 1165 (1972).

${ }^{17}$ C. Lopez, F. J. Yndurain, and N. Garcia, Phys. Rev. B 18, 970 (1978).

${ }^{18}$ G. A. Farias and A. A. Maradudin, Phys. Rev. B 28, 5675 (1983).

${ }^{19}$ A. A. Maradudin and W. M. Vissher, Z. Phys. B 60, 215 (1985).

${ }^{20}$ D. Maystre, thesis, Université Aix-Marseille III, Marseille, 1974.

${ }^{21}$ M. Neviere, P. Vincent, and R. Petit, Nouv. Rev. Opt. 5, 65 (1974).

${ }^{22}$ A. Wirgin, Opt. Acta 28, 1377 (1981). 